\title{
Impact of implementing the southampton physiotherapy post-operative screening tool (SPPOST)
}

\author{
J Weblin", F Williams, DJ McWilliams \\ From ESICM LIVES 2015 \\ Berlin, Germany. 3-7 October 2015
}

\begin{abstract}
Introduction
The benefit and necessity for prophylactic physiotherapy post operatively remains unclear [1]. Combined with an increased demand on resources, scores to identify patients who would most benefit are being increasingly used. A recent blinded observational evaluation of the SPPOST within our surgical physiotherapy service suggested it to be a potentially useful tool for identifying low risk patients who did not require physiotherapy intervention.
\end{abstract}

\section{Objectives}

To formally introduce the SPPOST and analyse the impact of withdrawing physiotherapy intervention for low risk surgical patients on a liver surgical ward.

\section{Methods}

All patients undergoing liver surgical procedures between $12^{\text {th }}$ July and $13^{\text {th }}$ August 2014 were included in the analysis. SPPOST scores were calculated on the first day postoperatively. For patients identified as low risk (Score $<10$ ) post-operative care and mobilisation was led by the nursing staff with no physiotherapy involvement. Patients identified as high risk received standard physiotherapy input, including respiratory interventions and mobilisation as required. Primary outcome was development of post-operative pulmonary complications (PPC) according to the BrooksBrunn criteria [2]. Secondary measures analysed were the number of low risk patients re-referred for physiotherapy, time to sit out of bed and time to walk $>30$ meters.

\section{Results}

57 patients who had undergone liver surgery were included in the analysis, of which 24 (42\%) were classified as low risk. None of the patients identified as low risk developed a PPC, although one low risk patient was rereferred to physiotherapy for upper limb exercises only. With regards to mobilisation, 20 (83\%) of the low risk patients sat out of bed on day 1 , whilst the remaining 4 (17\%) did so on day 2 , with no adverse events recorded. The median (IQR) time to walk 30 metres was 3 days [2-4]. Low risk patients spent on average 7.08 days in hospital, 1.47 less than the high risk group.

\section{Conclusions}

Our results confirm the SPPOST as an effective postoperative screening tool, with no patients identified as low risk developing a PPC. For these low risk patients, mobilisation was safely led by nursing staff with none re-referred for either pulmonary physiotherapy or mobility assistance. However, recent enhanced recovery guidelines provide a target of walking patients on the first post-operative day, although very few patients achieved this goal in our low risk patient group. Further education for both patients and the nursing staff around 'early mobilisation' may assist patients to meet this target.

Published: 1 October 2015

\section{References}

1. Pasquina P, Tramer MR, Granier J, Walder B: Respiratory physiotherapy to prevent pulmonary complications after abdominal surgery. Chest 2006, 130(6):1887-1889.

2. Brooks-Brunn JA: Predictors of post-operative pulmonary complications following abdominal surgery. Chest 1997, 111(3):564-571.

doi:10.1186/2197-425X-3-S1-A554

Cite this article as: Weblin et al:: Impact of implementing the southampton physiotherapy post-operative screening tool (SPPOST). Intensive Care Medicine Experimental 2015 3(Suppl 1):A554. 